\title{
BMJ
}

\section{Effect of preventive primary care outreach on health related quality of life among older adults at risk of functional decline: randomised controlled trial}

\author{
Jenny Ploeg, associate professor, ${ }^{1}$ Kevin Brazil, professor, ${ }^{2}$ director, ${ }^{3}$ Brian Hutchison, professor emeritus, ${ }^{4}$ \\ Janusz Kaczorowski, associate professor, ${ }^{5}$ Dawn M Dalby, assistant professor, ${ }^{6}$ Charles H Goldsmith, \\ professor emeritus, ${ }^{7,8}$ William Furlong, research associate ${ }^{9}$
}

${ }^{1}$ School of Nursing, Faculty of

Health Sciences, McMaster

University, 1200 Main St W, Room

HSc-3N28G Hamilton, ON, Canada

L8N $3 Z 5$

${ }^{2}$ Department of Clinical

Epidemiology and Biostatistics,

Division of Palliative Care,

Department of Family Medicine

Faculty of Health Sciences,

McMaster University

${ }^{3}$ St Joseph's Health System

Research Network, Hamilton, ON,

Canada L8N 1G6

${ }^{4}$ Departments of Family Medicine and Clinical Epidemiology and

Biostatistics and Centre for Health

Economics and Policy Analysis,

Faculty of Health Sciences,

McMaster University

${ }^{5}$ Department of Family Practice,

University of British Columbia,

Child and Family Research

Institute, Vancouver, BC, Canada

V6T 1 Z3

${ }^{6}$ Department of Kinesiology and Physical Education, Wilfrid Laurier University, Waterloo, ON, Canada N2L 3C5

${ }^{7}$ Department of Clinical

Epidemiology and Biostatistics, McMaster University

${ }^{8}$ Biostatistics Unit, St Joseph's Healthcare Hamilton, Hamilton,

ON, Canada L8N 4A6

${ }^{9}$ Health Utilities Group, Centre for Health Economics and Policy

Analysis, Department of Clinical

Epidemiology and Biostatistics,

McMaster University

Correspondence to: J Ploeg

ploegj@mcmaster.ca

Cite this as: $B M J$ 2010;340:c1480 doi:10.1136/bmj.c1480

\section{ABSTRACT}

Objective To evaluate the impact of a provider initiated primary care outreach intervention compared with usual care among older adults at risk of functional decline.

Design Randomised controlled trial.

Setting Patients enrolled with 35 family physicians in five primary care networks in Hamilton, Ontario, Canada.

Participants Patients were eligible if they were 75 years of age or older and were not receiving home care services. Of 3166 potentially eligible patients, 2662 (84\%) completed the validated postal questionnaire used to determine risk of functional decline. Of 1724 patients who met the risk criteria, 769 (45\%) agreed to participate and 719 were randomised.

Intervention The 12 month intervention, provided by experienced home care nurses in 2004-6, consisted of a comprehensive initial assessment using the resident assessment instrument for home care; collaborative care planning with patients, their families, and family physicians; health promotion; and referral to community health and social support services.

Main outcome measures Quality adjusted life years (QALYs), use and costs of health and social services, functional status, self rated health, and mortality.

Results The mean difference in QALYs between intervention and control patients during the study period was not statistically significant $(0.017,95 \%$ confidence interval -0.022 to $0.056 ; \mathrm{P}=0.388$ ). The mean difference in overall cost of prescription drugs and services between the intervention and control groups was not statistically significant, (-\$C165 (£107; €118; \$162), 95\% confidence interval -\$C16 545 to $\$ C 16214 ; \mathrm{P}=0.984$ ). Changes over 12 months in functional status and self rated health were not significantly different between the intervention and control groups. Ten patients died in each group.

Conclusions The results of this study do not support adoption of this preventive primary care intervention for this target population of high risk older adults.

Trial registration Clinical trials NCT00134836.

\section{INTRODUCTION}

The provision of high quality, comprehensive care for older adults is becoming increasingly challenging because of the ageing of society, shortages of healthcare providers, and rising healthcare costs. ${ }^{12}$ These changes call for the development and evaluation of practical and cost effective approaches to care for older adults. Systematic reviews of interventions such as health assessments, home based support, geriatric evaluation and management, home based nursing health promotion, home visits, and preventive home visits attest to the different approaches evaluated to date. ${ }^{3-8}$ The findings from these systematic reviews have been inconsistent - some have reported benefits, some have found no evidence of impact, and others have had mixed findings.

The evidence suggests that interventions such as geriatric evaluation and management should be targeted at specific groups of older adults most likely to benefit. ${ }^{9}$ Recognised criteria for targeting older adults for such interventions include advanced age, degree of functional impairment, and presence of other conditions. ${ }^{10}$ However, one systematic review of home visits to reduce functional decline found that survival benefits were seen in young-old (72.7-77.5 years) rather than old-old (80.2-81.6 years) populations. ${ }^{7}$ Further research is needed to determine which components of preventive home visits work best in which groups of older adults. ${ }^{11}$

This study evaluated one approach to caring for older adults-preventive primary care outreach. ${ }^{12}$ We define preventive primary care outreach as proactive, provider initiated care above and beyond demand led routine care, provided in a community primary care setting, and linked to the usual care system. ${ }^{12}$ Its goals are to identify unrecognised problems and people at increased risk and to link those people to appropriate health and social care. Preventive primary care outreach involves an initial comprehensive assessment and individualised follow-up. A preventive approach based on identifying people at risk and providing early intervention might help to prevent or delay functional 


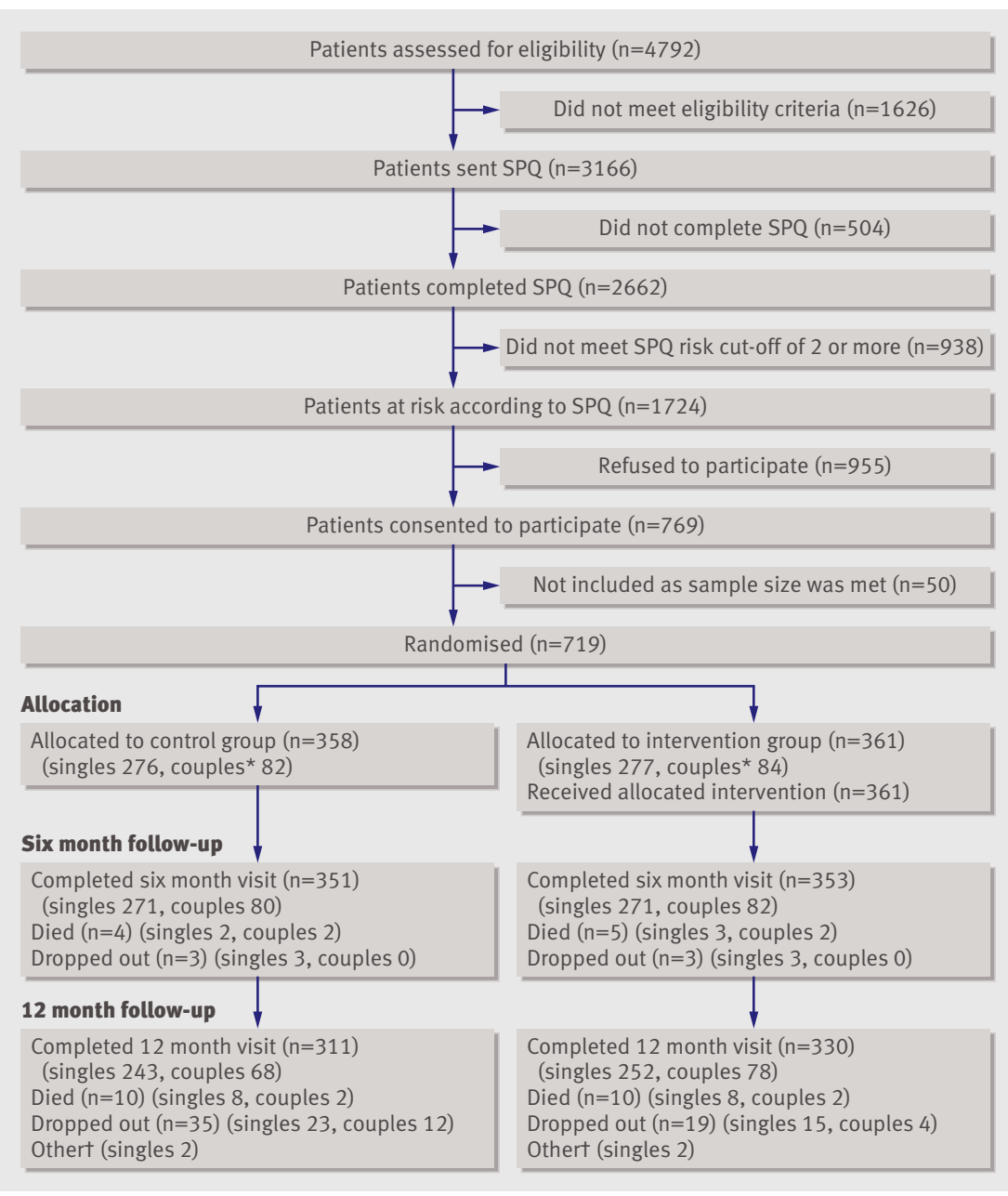

Flow diagram of trial. SPQ=Sherbrooke postal questionnaire. *Numbers refer to individual patients (for example, $\mathrm{n}=82$ refers to 41 couples). $\dagger$ Two patients in each group died after 12 month visit but did not have 12 month assessment; their missing data were imputed in analysis with control patients would have more quality adjusted life years, higher functional status and self rated health, lower mortality, and similar costs of health and social services.

The intervention was provided to patients of family physicians who were members of primary care networks in Hamilton, Ontario, Canada. Primary care networks comprise networks of solo and small group practices of family physicians. ${ }^{15}$ They differ from the dominant fee for service model in Ontario by virtue of enrolling patients and remunerating physicians through a capitation based blended payment model that includes fees for preventive care outreach, home care supervision, and team consultation and bonus payments for achievement of target levels of influenza vaccination of seniors, cervical smears, mammography, and childhood immunisation.

We chose primary care networks for this study because they had computerised lists of enrolled patients and provided primary care to approximately half of the community's population at the time of the study. In 2007, primary care networks and health service organisations (another capitation based model) were harmonised to become family health organisations.

\section{METHODS}

We used a three stage process to recruit patients: random selection of primary care networks until the required sample size was attained, approaching all family physicians within the selected primary care network to participate in the study, and screening of patients who were enrolled with participating family physicians for their risk of functional decline.

\section{Recruitment of family physician practices}

We randomly selected five of eight primary care networks to participate in the study. As primary care networks were selected, we asked all family physicians within the primary care network to participate in the study. Two family physicians who were well known and respected by their colleagues in the community assisted with recruitment. Research assistants worked with staff in the participating physicians' offices to identify patients who met the study inclusion criteria.

\section{Screening and recruitment of patients}

We used the Sherbrooke postal questionnaire to screen patients for risk of functional decline. ${ }^{16}$ This questionnaire includes six items related to mobility, vision, hearing, memory, drug use, and living alone. Each item with a positive response is given a score of 1 , with a minimum scale score of 0 and a maximum of 6 . In a similar population, the Sherbrooke postal questionnaire was shown to have $75 \%$ sensitivity, $52 \%$ specificity, and a positive predictive value of $38 \%$ for functional decline. ${ }^{16}$ Validity was tested by comparing scores on the initial 29 item instrument with the nursing time required for care $(\mathrm{r}=0.88) .{ }^{16} \mathrm{In}$ our study, we mailed the Sherbrooke postal questionnaire with a covering letter from the patient's family physician followed by up to two mailed reminders. We identified 
Table 1|Patients' characteristics at baseline. Values are numbers (percentages) unless stated otherwise

\begin{tabular}{|c|c|c|}
\hline Characteristics & Intervention $(\mathrm{n}=361)$ & Control $(n=358)$ \\
\hline Mean (SD) age (years) & $81.0(4.1)$ & $81.3(4.4)$ \\
\hline Female sex & $188(52)$ & $194(54)$ \\
\hline \multicolumn{3}{|l|}{ Marital status: } \\
\hline Married, common law & $203(56)$ & $197(55)$ \\
\hline Widowed & $133(37)$ & $124(35)$ \\
\hline Separated, divorced & $10(3)$ & $24(7)$ \\
\hline Single & $14(4)$ & $13(4)$ \\
\hline Not answered & 1 & 0 \\
\hline \multicolumn{3}{|l|}{ Education: } \\
\hline Less than primary school & $22(6)$ & $19(5)$ \\
\hline Completed primary school & $70(19)$ & $83(23)$ \\
\hline Some secondary school & $109(30)$ & $96(27)$ \\
\hline Completed secondary school & $76(21)$ & $71(20)$ \\
\hline Some community college & $17(5)$ & $21(6)$ \\
\hline Completed community college & $24(7)$ & $28(8)$ \\
\hline Some university & $16(4)$ & $14(4)$ \\
\hline University degree & $27(8)$ & $26(7)$ \\
\hline \multicolumn{3}{|l|}{ Household income: } \\
\hline Below $\$ 20000$ & $74(21)$ & $74(21)$ \\
\hline$\$ 20000-\$ 39999$ & $163(45)$ & $178(50)$ \\
\hline$\$ 40000-\$ 59999$ & $50(14)$ & $49(14)$ \\
\hline$\$ 60000-\$ 79999$ & $17(5)$ & $11(3)$ \\
\hline$\$ 80000-\$ 99999$ & $3(1)$ & $7(2)$ \\
\hline Over $\$ 100000$ & $12(3)$ & $9(3)$ \\
\hline Refused to answer/missing & $42(12)$ & $30(8)$ \\
\hline Live alone & $119(33)$ & $125(35)$ \\
\hline Born in Canada & $213(59)$ & $220(61)$ \\
\hline \multicolumn{3}{|c|}{ Standardised mini-mental state exam score (out of 30 ): } \\
\hline Normal (26-30) & $283(78)$ & $288(80)$ \\
\hline Mild cognitive impairment (20-25) & $73(20)$ & $66(18)$ \\
\hline Moderate cognitive impairment (10-19) & $4(1)$ & $4(1)$ \\
\hline Mean (SD) Health Utilities Index Mark 3 & $0.54(0.31)$ & $0.51(0.32)$ \\
\hline Missing & 13 & 17 \\
\hline
\end{tabular}

Scores on Sherbrooke postal

questionnaire:

\begin{tabular}{lcc}
\hline 2 & $147(41)$ & $145(41)$ \\
\hline 3 & $122(34)$ & $105(29)$ \\
\hline 4 & $55(15)$ & $69(19)$ \\
\hline 5 & $31(9)$ & $28(8)$ \\
\hline 6 & $6(2)$ & $11(3)$ \\
\hline
\end{tabular}

patients who provided a positive response to at least two questions (score of 2 or more) as being at risk of functional decline, and their family physician mailed them a letter inviting them to participate in the study.

\section{Study participants}

Eligible patients were aged 75 years or older, they or their proxy were able to answer questions in English, and they resided in the city of Hamilton, Ontario, Canada. Patients were ineligible if they received home care services, lived in a nursing home or long term care home, were identified by their family physician as needing palliative care, were scheduled for major elective surgery in the next year, or were planning to leave the country for more than one month during the 12 month follow-up period.

\section{Study design}

The study was a randomised controlled trial with an intervention group and a control group receiving usual care. Research staff assessed patients for eligibility and informed the statistician (CHG) about eligible patients to be randomised. We randomised each couple as a cluster of two and single people as individuals. We used a 1:1 allocation ratio to allocate individuals or couples to either the intervention group or the control group. The sequence of allocations was generated in blocks of eight or 16 such that the allocation was balanced in blocks. The random numbers used to assign the block size and choice of allocation within blocks came from the Rand tables of random digits. ${ }^{17}$ The allocation sequence was kept in the locked office of the statistician and was inaccessible to staff making decisions about patients' eligibility. The results of the allocation were communicated to the person who arranged for the nurses to visit the appropriate patients but not to the research assistants collecting outcome assessments. Research assistants were thus blinded to group assignment.

Any single patient who was a partner of an already allocated patient was assigned to the same group as the first recruited partner of that couple. We defined study groups for analyses according to the intention to treat principle. Participants gave written informed consent before taking part in the study.

\section{Preventive primary care outreach intervention}

The 12 month intervention consisted of a comprehensive initial assessment, collaborative care planning, health promotion, and referral to community health and social support services. Three experienced home care nurses delivered the intervention by using the resident assessment instrument for home care system, composed of the minimum dataset for home care and the client assessment protocols. ${ }^{18}$ Particular items in the minimum dataset for home care trigger up to 30 client assessment protocols that identify patients who could benefit from further evaluation and intervention. The client assessment protocols include guidelines that the nurses used for further assessment and care planning. Patients' assessments were completed in their homes at baseline and at six and 12 months and triggered new interventions and recommendations at each assessment.

Nurses encouraged patients to take an active part in their health care and worked closely with patients and their families. Health promotion materials given to and discussed with patients covered safety in the home including falls prevention, safe drug management, nutrition, upper and lower body strengthening exercises, colorectal screening, and influenza vaccination. Nurses provided health education on topics such as management of chronic diseases (for example, diabetes and heart disease) and encouraged the use of calcium and vitamin D supplements. Where appropriate, 
Table 2|Patients' outcomes (without multiple imputation of missing values)

\begin{tabular}{|c|c|c|c|c|c|c|}
\hline \multirow[b]{2}{*}{ Outcome } & \multicolumn{3}{|c|}{ Intervention } & \multicolumn{3}{|c|}{ Control } \\
\hline & Mean (SD) & Median (IQR) & No & Mean (SD) & Median (IQR) & No \\
\hline Quality adjusted life years (QALYs)* & $0.5554(0.2621)$ & $0.5876(0.3557-0.7495)$ & 287 & $0.5079(0.2820)$ & $0.5465(0.2966-0.7368)$ & 267 \\
\hline Cost of prescription drugs† & $2440(2977)$ & $1581(927-2601)$ & 309 & $2563(3016)$ & $1633(972-2876)$ & 308 \\
\hline Cost of services & $5469(7330)$ & 3401 (1898-6188) & 361 & $5795(8758)$ & $3126(1588-6460)$ & 343 \\
\hline $\begin{array}{l}\text { Combined costs of prescription drugs } \\
\text { and servicest }\end{array}$ & 7779 (7980) & $5558(3426-8630)$ & 350 & $8096(9582)$ & 5089 (3174-9519) & 343 \\
\hline $\begin{array}{l}\text { Older Americans resources and services } \\
\text { multidimensional functional assessment- } \\
\text { activities of daily living } ₫ \S\end{array}$ & $0.207(0.922)$ & $0(0.000-1.000)$ & 328 & $0.145(0.817)$ & $0(0.000-1.000)$ & 310 \\
\hline Self rated health§ণ & $0.031(0.856)$ & $0(-1.000-1.000)$ & 328 & $0.068(0.955)$ & $0(0.000-1.000)$ & 310 \\
\hline
\end{tabular}

$\mathrm{IQR}=$ interquartile range.

*High score is good.

†CAN including intervention costs for intervention group.

†High score is good.

$\$ 12$ month minus baseline.

ILow score is good.

nurses discussed stress on caregivers and options for its relief including nursing home placement. In negotiation with patients and their families, referrals were made to various community health and support services such as home care services, meals on wheels, and outpatient clinics. In some cases, patients were assisted in obtaining bathroom equipment and mobility aids. After each visit, nurses left a card in the home outlining their interventions and any actions required by the patient, such as follow-up with their own family physician. Nurses monitored and encouraged patients' adherence to their recommendations through followup phone calls and home visits.

After each home visit, nurses faxed a physician communication form to the patient's family physician. This form outlined the client assessment protocols that were triggered at the visit, nursing actions taken to tackle any problems, and areas of follow-up required by the physician. Nurses worked closely with the physician and other professionals (such as pharmacist, dietitian, and physiotherapist) to implement the plan of care.

\section{Outcome measures}

The primary outcome measure was quality adjusted life years (QALYs) with Health Utilities Index Mark 3 health related quality of life utility scores as the quality adjustment weights. ${ }^{19-25}$ The Health Utilities Index Mark 3 is a reliable and valid generic system for measuring comprehensive health status and overall health related quality of life. ${ }^{2326}$ It consists of two basic components: a comprehensive classification system for health status and a utility scoring function. The health status classification system consists of eight attributes (vision, hearing, speech, ambulation, dexterity, emotion, cognition, and pain/discomfort), each with five to six levels, and describes 972000 unique health states. The utility scoring function provides a utility score of health related quality of life for each unique health state. The utility scores represent measurements of community preferences as recommended by many national guidelines for calculating QALYs for use in cost effectiveness analyses. ${ }^{26}$
We chose the QALY as the primary outcome measure because it integrates two major health concerns in the study population: morbidity and mortality. The Health Utilities Index Mark 3 comprehensively measures morbidity across eight attributes/dimensions of health. Three other intervention studies (two in Canada and one in the United States) have used health related quality of life or QALYs (using the Health Utilities Index Mark 3) as a primary or secondary outcome measure. ${ }^{27-29}$ Two of these studies found a statistically significant difference between intervention and control groups. ${ }^{2829}$

The Health Utilities Index Mark 3 questionnaire asked each respondent about the patient's health status during a two week recall period. The utility scale for health related quality of life is defined such that being dead has a score of 0.00 , perfect health has the maximum score of 1.00 , negative scores are associated with states considered to be worse than being dead, and the minimum score is -0.36 . Patients who died during the study period were assigned a utility score of 0.00 for all subsequent assessment points. To place the baseline scores of patients in our intervention group (0.54) and control group (0.51) in context, they can be compared with the following scores: 0.90 for the general adult population (mean age 38 years), ${ }^{30} 0.75$ for the general older adult population (age 75-89), ${ }^{31} 0.60$ for adults (mean age 66 years) with diabetes, ${ }^{32}$ and 0.50 for adults (mean age 63 years) with knee osteoarthritis. ${ }^{28}$

Secondary outcome measures included costs of health and social services, functional status, self rated health, and mortality. We included the nursing costs to deliver the intervention in the total costs. The measurement of costs was based on the quantities and unit costs of health and social services used by each patient. We used the health and social service utilization survey to collect data. ${ }^{33}$ This survey includes items related to a comprehensive array of service costs (for example, visits to the family physician, hospital admissions, and home nursing visits) as well as medical procedures and prescription drugs. Participants were asked to indicate the number of times they had used each type of 
Table 3|Patients' outcomes, with multiple imputation

\begin{tabular}{|c|c|c|c|}
\hline Outcome & $\begin{array}{l}\text { Difference (intervention } \\
\text { minus control) }(95 \% \mathrm{Cl})\end{array}$ & $P$ value & Intraclass correlation \\
\hline Quality adjusted life years (QALYs)* & $0.017(-0.022$ to 0.056$)$ & 0.388 & 0.297 \\
\hline Cost of prescription drugs† & $-65(-5849$ to 5719$)$ & 0.982 & 0.000 \\
\hline Cost of services $†$ & $-100(-14920$ to 14720$)$ & 0.989 & 0.049 \\
\hline Combined costs of prescription drugs and services $†$ & $-165(-16545$ to 16214$)$ & 0.984 & 0.009 \\
\hline $\begin{array}{l}\text { Older Americans resources and services multidimensional functional } \\
\text { assessment-activities of daily living } ₫ \S\end{array}$ & $0.091(-0.042$ to 0.223$)$ & 0.180 & 0.280 \\
\hline Self rated health§ा & $-0.015(-0.158$ to 0.127$)$ & 0.832 & 0.110 \\
\hline
\end{tabular}

*High score is good (positive difference estimate favours intervention).

†\$CAN including intervention costs for intervention group (negative difference estimate favours intervention).

$\ddagger$ High score is good (positive difference estimate favours intervention).

$\$ 12$ month value minus baseline value.

ILow score is good (negative difference estimate favours intervention).

resource during the previous six months (hospital admission, emergency visit, admission to long term care facility), the previous two weeks (other health and social services), or the previous four days (prescription drugs and special treatments). We measured functional status by self report with the five items in the activities of daily living section of the older Americans resources and services multidimensional functional assessment. ${ }^{34}$ The scoring scale of this measure varies from a minimum of 5 to a maximum of 15 ; a lower score indicates more impaired functional abilities. We assessed self rated health by using a single item from the medical outcomes study SF-36, asking respondents to rate their health on a five category scale from excellent (1) to poor (5). ${ }^{3536}$

All outcome measures were assessed at baseline and 12 months. Health Utilities Index Mark 3 and health and social service utilization survey measurements were also collected at six months. Research assistants made home visits to collect outcome data. When patients had died before the next follow-up visit by the research assistant, the research assistant obtained the date of death by contacting the family physician or a family member. Outcome measurements were collected between 26 August 2004 and 26 June 2006.

\section{Sample size}

Health Utilities Index Mark 3 scores are available for 7600 respondents to the 2000-1 Canadian community health survey aged 75 years or older. Anticipating that the Sherbrooke postal questionnaire would identify approximately $50 \%$ of those screened for our study as being at risk of functional decline, we used the mean (0.486) and standard deviation (0.277) of Health Utilities Index scores among the Canadian community health survey respondents 75 years of age or older whose scores were at or below the median to estimate the initial Health Utilities Index scores in our sample. We used data from the 2000-1 Canadian community health survey and the 1990 Ontario health survey to estimate the expected decline in Health Utilities Index scores over 15 months due to ageing (0.02). (The sample size was originally calculated for a 15 month intervention, but we were only able to offer a
12 month intervention.) We estimated the expected survival rate among control group participants at 15 months' follow-up to be $91 \%$ on the basis of data from the control arms of randomised controlled trials in the meta-analysis of preventive primary care outreach interventions. ${ }^{12}$ Combining these data, we calculated the expected end of study mean Health Utilities Index score among control participants to be 0.424 . Given the $20 \%$ reduction in mortality attributable to preventive primary care outreach interventions at one year follow-up in our meta-analysis, we anticipated that the mean Health Utilities Index score at 15 months among intervention patients would be at least 15\% higher than that among control group patients. On the basis of an average loss to follow-up of $7.2 \%$ in the preventive primary care outreach trials in the meta-analysis, we conservatively assumed a $10 \%$ loss to follow-up for our sample size calculations. Setting $\alpha$ at 0.05 and $\beta$ at 0.2 , we needed to recruit 664 patients (332 in each group). ${ }^{37}$

\section{Analysis}

We used the Health Utilities Index Mark 3 scores of health related quality of life at baseline, six months, and 12 months to calculate QALYs, using the area under the curve technique. The health related quality of life curve consists of horizontal segments for the two week health status recall periods and straight lines joining these horizontal segments (that is, a trapezoid estimation rule). When a patient died between assessment times, we assigned a health related quality of life score of 0.00 from the date of death and used a straight line to join the previous health related quality of life score and this 0.00 . For the outcomes of self rated health and older Americans resources and services multidimensional functional assessment-activities of daily living, we calculated the change in scores (12 month value minus baseline value) for the intervention and control groups.

We based unit costs for each service and prescription drug used on means for the province of Ontario, Canada. ${ }^{3839}$ We described use of services in the intervention and control groups by using descriptive statistics (mean, standard deviation, interquartile range) and 
Table $4 \mid$ Number of visits per patient without multiple imputation of missing values

\begin{tabular}{|c|c|c|c|c|c|c|c|}
\hline \multirow[b]{2}{*}{ Visits } & \multicolumn{3}{|c|}{ Intervention } & \multicolumn{3}{|c|}{ Control } & \multirow[b]{2}{*}{ Pvalue (KS; Kuiper)* } \\
\hline & Mean (SD) & Median (IQR) & No & Mean (SD) & Median (IQR) & No & \\
\hline Family physician† & 7.93 (10.49) & $0(0-13)$ & 331 & $8.51(10.43)$ & $0(0-13)$ & 313 & $0.897 ; 0.989$ \\
\hline Hospital admissions & $0.29(0.66)$ & $0(0-0)$ & 331 & $0.49(1.50)$ & $0(0-1)$ & 311 & $0.893 ; 1.00$ \\
\hline Emergency room & $0.44(0.90)$ & $0(0-1)$ & 331 & $0.66(1.19)$ & $0(0-0)$ & 312 & $0.085 ; 0.452$ \\
\hline Nursing† & $5.04(23.80)$ & $0(0-0)$ & 330 & $2.68(22.20)$ & $0(0-0)$ & 310 & $0.658 ; 0.986$ \\
\hline Specialist† & $6.58(11.12)$ & $0(0-13)$ & 330 & $6.29(10.75)$ & $0(0-13)$ & 310 & $1.000 ; 1.000$ \\
\hline Physiotherapy & $2.72(12.01)$ & $0(0-0)$ & 330 & 3.15 (13.89) & $0(0-0)$ & 310 & $1.000 ; 1.000$ \\
\hline Occupational therapy & $0.83(4.84)$ & $0(0-0)$ & 330 & $0.34(3.45)$ & $0(0-0)$ & 310 & $0.999 ; 1.000$ \\
\hline Social worker† & $0.24(2.25)$ & $0(0-0)$ & 330 & $0.46(3.96)$ & $0(0-0)$ & 310 & $1.000 ; 1.000$ \\
\hline Nutritionist† & $0.39(2.65)$ & $0(0-0)$ & 330 & $0.34(2.07)$ & $0(0-0)$ & 310 & $1.000 ; 1.000$ \\
\hline Homemaker† & $3.62(23.25)$ & $0(0-0)$ & 330 & 3.73 (23.89) & $0(0-0)$ & 310 & $1.000 ; 1.000$ \\
\hline $\begin{array}{l}\text { No }(\%) \text { patients with long } \\
\text { term care admission }\end{array}$ & & 331 (2.1) & & & 314 (3.5) & & $0.343 \ddagger$ \\
\hline
\end{tabular}

$\mathrm{IQR}=$ interquartile range.

*Because distributions are heavily skewed and assumption of normality was violated, two non-parametric tests were used to compare intervention and control groups: Kolmorgorov-Smirnov (KS) test and Kuiper test.

†Values estimated on basis of reported visits during previous two weeks at six month and 12 month assessments.

$\ddagger$ Fisher's exact test.

compared them by using both the KolmogorovSmirnov test and the Kuiper test, the first of which generally has the lower power. ${ }^{40}$ We used a two tailed Fisher exact test to compare the number of patients with a long term care admission. We calculated the total daily costs of all service and prescription drug items for each patient at baseline (cost 1), six months (cost 2), and 12 months (cost 3). We used the formula $((\cos t 1+\operatorname{cost} 2) / 2) \times 182+((\operatorname{cost} 2+\operatorname{cost} 3) / 2) \times 183$ to calculate annual costs for each patient who completed the study to 365 days. We made adjustments to the calculation on the basis of date of dropout and missing data.

We used simple descriptive statistics (mean, median, standard deviation, interquartile range) to describe the primary and secondary outcomes for the intervention and control groups. The estimates of effect for QALYs, functional status, self rated health, and costs of prescription drugs and services were the difference between intervention and control groups. We used analysis of variance to calculate the $95 \%$ confidence intervals and $\mathrm{P}$ values for these estimates, recognising the clustering and blocking in the design, as well as 10 imputations to handle missing data and dropouts from the study apart from death.

We set statistical significance at the $5 \%$ level. Because of the clustering effect of random allocation by households, we estimated the intraclass correlations in keeping with the recommendations of Donner and Klar. ${ }^{41}$ We used SAS software version 9.2 for all analyses.

\section{Multiple imputation analyses}

We used multiple imputation to fill in missing data. ${ }^{42}$ We used the following variables that had few missing data points to begin the imputation process: sex, marital status, educational level, baseline self rated health, randomisation date, six month assessment date, and 12 month assessment date. We created 10 complete datasets and analysed each separately. We used the SAS software procedure PROC MIANALYSE to combine the results of these analyses.

\section{Subgroup analysis}

Because we were interested in exploring the differential effect of the intervention on patients at higher compared with lower risk of functional decline, we did a post hoc analysis (using two way analysis of variance and its $\mathrm{F}$ tests) of differences in outcomes for patients who were at greater risk of functional decline (Sherbrooke postal questionnaire scores of 4-6) compared with those who were at lower risk of functional decline (scores of 2-3).

\section{RESULTS}

\section{Participant characteristics}

Thirty-five (47\%) of 74 family physicians agreed to participate. Of the 4792 patients aged 75 years and over enrolled with participating physicians, 1626 did not meet the inclusion criteria and were not sent the Sherbrooke postal questionnaire (figure). Of the eligible patients who were sent the questionnaire, 504/3166 (15.9\%) did not complete it. Of the 2662 patients who completed the questionnaire, $938(35.2 \%)$ scored below the cut-off score of 2 for risk and were not eligible to participate. Of the 1724 patients who were eligible, $955(55.4 \%)$ refused to participate. Between 5 August 2004 and 31 May 2005, we randomised 719 patients. Of the 719 randomised patients, 641 (89.2\%) were assessed at 12 months. Characteristics of patients in the intervention and control groups were similar (table 1). The follow-up rate at 12 months was $91 \%$ (330/361) in the intervention group and 87\% (311/ $358)$ in the control group.

\section{Intervention}

The number and frequency of home visits varied according to the needs of each patient. The mean number of home visits per patient was 3.03 (minimum 1; maximum 7), indicating that most patients received the planned three home visits over the year. Patients received a mean of 1.17 telephone calls from the 
Table 5 Baseline and 12 month scores for self rated health item from SF-36 and older Americans resources and services multidimensional functional assessment-activities of daily living. Values are numbers (percentages)

\begin{tabular}{|c|c|c|c|c|}
\hline & \multicolumn{2}{|c|}{ Baseline } & \multicolumn{2}{|c|}{12 month } \\
\hline & Control $(n=358)$ & Intervention $(n=361)$ & Control $(n=310)$ & Intervention $(\mathrm{n}=328)$ \\
\hline \multicolumn{5}{|c|}{ Self rated health item from SF-36 } \\
\hline Excellent & $24(7)$ & $21(6)$ & $20(6)$ & $16(5)$ \\
\hline Very good & $81(23)$ & 69 (19) & $66(21)$ & $65(20)$ \\
\hline Good & $125(35)$ & $140(39)$ & $120(39)$ & $130(40)$ \\
\hline Fair & $93(26)$ & $112(31)$ & $72(23)$ & $99(30)$ \\
\hline Poor & $35(10)$ & $19(5)$ & $32(10)$ & $18(5)$ \\
\hline \multicolumn{5}{|c|}{ Older Americans resources and services multidimensional functional assessment-activities of daily living } \\
\hline Excellent-good & $81(23)$ & $75(21)$ & $71(23)$ & $66(20)$ \\
\hline Mild impairment & $156(44)$ & $171(47)$ & $114(37)$ & $121(37)$ \\
\hline Moderate impairment & $87(24)$ & $78(22)$ & $86(28)$ & $90(27)$ \\
\hline Severe impairment & $21(6)$ & $23(6)$ & $23(7)$ & $28(9)$ \\
\hline Total impairment & $13(4)$ & $14(4)$ & $16(5)$ & $23(7)$ \\
\hline
\end{tabular}

nurse in addition to the home visits over the year. Referrals were most often made to the regional home care agency and the family physician.

\section{Primary outcome measure}

The mean QALYs for the intervention and control group, without multiple imputation, were 0.5554 (SD 0.2621 ) and 0.5079 (SD 0.2820). Tables 2 and 3 show outcomes without and with imputation of missing values. The difference in QALYs (intervention group minus control group), with multiple imputation, was not statistically significant $(0.017,95 \%$ confidence interval -0.022 to $0.056 ; \mathrm{P}=0.388)$.

The mean unimputed Health Utilities Index Mark 3 scores for intervention and control groups from which QALYs were derived were as follows. Baseline scores were 0.54 (SD 0.31, $\mathrm{n}=348$ ) in the intervention group and 0.51 (SD 0.32, $\mathrm{n}=341$ ) in the control group. Six month scores were 0.55 (SD $0.31, \mathrm{n}=327$ ) in the intervention group and $0.50(\mathrm{SD} 0.32, \mathrm{n}=316)$ in the control group. Twelve month scores were 0.49 (SD 0.31, $\mathrm{n}=318$ ) in the intervention group and 0.47 (SD 0.32, $\mathrm{n}=302$ ) in the control group.

\section{Secondary outcome measures}

We found no statistically significant differences between the two groups in the costs of prescription drugs $(\mathrm{P}=0.982)$, health and social services $(\mathrm{P}=0.989)$, or prescription drugs and health and social services combined $(\mathrm{P}=0.984)$. The mean total cost for services and prescription drugs (table 3 ) used by the intervention group over the year was approximately \$C165 (£107; €118; \$162) less than that for the control group; the data were highly skewed. The mean, standard deviation, and interquartile range of visits to the family physician, physician specialist, emergency room visits, and hospital admissions were fairly similar in the two groups (table 4). Similarly, the number of visits for physiotherapy and occupational therapy and visits by social workers, nutritionists, and homemakers were similar in the two groups. The number of patients who were admitted to a long term care home was similar in the intervention $(2.1 \%)$ and control $(3.5 \%)$ groups.

Table 5 shows baseline and 12 month scores for self rated health and functional status by group. We found no statistically significant differences in change ( 12 month minus baseline) in functional status $(\mathrm{P}=0.180)$ or self rated health $(\mathrm{P}=0.832)$ between the intervention and control groups (table 3 ). Ten patients in each group died during the 12 month study period.

We found a QALY difference of 0.017 in our study, and given our actual sample size we had a power of 0.11 to detect a difference of that magnitude. The observed QALY difference of 0.017 is about $27 \%$ of the effect we anticipated of 0.064 .

\section{Subgroup analysis}

We did analyses to assess for a differential effect of the intervention on patients at higher compared with lower risk of functional decline. Table 6 shows the mean, median, standard deviation, and interquartile range of QALYs for patients who were at higher risk of functional decline (Sherbrooke postal questionnaire scores 4-6) and those who were at lower risk of functional decline (scores 2-3) in each group. The results of an analysis of variance (table 7 ) show that no interaction existed between the risk groups and the treatment effect $(\mathrm{P}=0.671)$, no overall treatment effect occurred $(\mathrm{P}=0.989)$, and the low risk group had higher QALYs than did the high risk group $(\mathrm{P}=0.003)$. However, we found no significant treatment effect for either the low risk group $(\mathrm{P}=0.291)$ or the high risk group $(\mathrm{P}=0.565)$.

\section{DISCUSSION}

In this study we found no effect of a preventive primary care outreach intervention for older adults at risk of functional decline on QALYs, costs of health and social services, functional status, self rated health, or mortality. This is a cost neutral intervention that may produce, at most, small health benefits. We found no differential effect of the intervention on QALYs for patients at higher or lower risk of functional decline. 
Table 6|HUI-3 QALYs by Sherbrooke postal questionnaire risk score and comparison group

\begin{tabular}{|c|c|c|c|c|c|c|}
\hline \multirow{2}{*}{$\begin{array}{l}\text { Sherbrooke postal questionnaire } \\
\text { risk score }\end{array}$} & \multicolumn{3}{|c|}{ Intervention } & \multicolumn{3}{|c|}{ Control } \\
\hline & Mean (SD) & Median (IQR) & No & Mean (SD) & Median (IQR) & No \\
\hline Low (scores 2-3) & $0.598(0.242)$ & $0.624(0.449-0.779)$ & 224 & $0.571(0.258)$ & $0.597(0.406-0.779)$ & 193 \\
\hline High (scores 4-6) & $0.404(0.276)$ & $0.440(0.148-0.627)$ & 63 & $0.342(0.277)$ & $0.337(0.149-0.552)$ & 74 \\
\hline
\end{tabular}

$\mathrm{IQR}=$ interquartile range.

\section{Study strengths and limitations}

Our study has several strengths that overcome the limitations of previous studies of such interventions. We used a validated postal questionnaire to determine risk of functional decline and included only participants aged 75 years and over who were at risk of functional decline. We assessed QALYs with an internationally used and well validated tool and assessed the use and cost of health and social services three times. We used a comprehensive and widely used assessment tool, minimum dataset for home care, as part of the intervention. Participants who were randomised had a high completion rate of $89 \%$ overall, $87 \%$ in the intervention group, and $91 \%$ in the control group. The distribution of potential confounding variables was similar between groups. The inclusion of intraclass correlations in our results may be valuable for planners of future studies with similar outcome measures in mixtures of older couples and singles.

Of eligible patients who completed the Sherbrooke postal questionnaire and were assessed to be at risk of functional decline, $55 \%$ did not agree to participate in the study. We do not know if these patients who refused to participate were similar to the participants for the variables assessed. Our sample size calculation was based on an expected annual mortality of $9 \%$ in the control group, and mortality was $2.8 \%$ in our study. Because the baseline Health Utilities Index Mark 3 scores were higher than anticipated and showed a smaller than expected decline over the 12 months of the study, the ability of patients to benefit from the study intervention may have been reduced. The variance inflation on QALYs was relatively high (30\%) owing to clustering (intraclass correlation $=0.297$ in table 3). Given that the mortality was lower than expected and the variance inflation on QALYs was larger than expected, we had less power than anticipated to rule out small but potentially important differences in quality adjusted length of life. The mean difference in QALYs between the intervention and control groups in our study, 0.017 , is equivalent to six

Table 7| Subgroup analysis of QALYs by high and low risk subgroups on the Sherbrooke postal questionnaire

\begin{tabular}{lcc} 
Effect & Estimate $(95 \% \mathrm{Cl})$ & P value \\
Treatment & $0.000(-0.068$ to 0.067$)$ & 0.989 \\
\hline Sherbrooke & $-0.214(-0.353$ to -0.075$)$ & 0.003 \\
\hline Treatment*Sherbrooke interaction & $0.047(-0.170$ to 0.263$)$ & 0.671 \\
\hline Treatment (for low risk Sherbrooke) & $0.023(-0.020$ to 0.067$)$ & 0.291 \\
\hline Treatment (for high risk Sherbrooke) & $0.023(-0.055$ to 0.100$)$ & 0.565
\end{tabular}

Treatment=intervention minus control; Sherbrooke=high minus low; analyses used were analysis of variance and its $\mathrm{F}$ tests. quality adjusted life days. We collected data related to use and costs of health and social services indirectly through reporting by patients.

\section{Comparison with previous studies}

Given the inconsistent results from published randomised controlled trials of similar interventions, our results are both consistent and inconsistent with previous literature. Our results are consistent with Canadian studies of similar interventions that found no differences between intervention and control groups in mortality, ${ }^{43-46}$ functional ability or decline, ${ }^{44-46}$ admission to an institution, ${ }^{43-45}$ health service use, ${ }^{43}$ and expenditures. ${ }^{46}$ Our results are also consistent with systematic reviews of randomised controlled trials of preventive home visits to older adults that found no difference in mortality, ${ }^{8}$ functional ability or decline, ${ }^{4}$ and admission to hospital. ${ }^{4}$

Our results were in contrast to other reviews of randomised controlled trials of similar interventions that found lowered mortality, fewer admissions to long term care, and increased likelihood of living in the community in the intervention group compared with the control group. ${ }^{42}$ Although the differences in findings may have been due to the shorter or less intense intervention of three home visits over one year or the inclusion of patients who were perhaps more healthy, the literature is not consistent with this interpretation. A systematic review of seven intensive home visiting programmes (at least four visits a year for at least 12 months) for older people with poor health status found that they were not beneficial in the healthcare settings of Western countries. ${ }^{47}$

A recent Canadian study of three payment models for primary care physicians (primary care networks, community health centres, and fee for service) in the management of hypertension found no significant dif ferences between the three models of care in physicians' sex or mean age, urban versus rural practice, academic versus community practice, and mean number of physicians per practice. ${ }^{48}$ No statistically significant differences existed between the three models of care in patients' age, sex, and socioeconomic status. Although the mean rate of screening for hypertension was similar across models of care, the mean rate of treatment was higher in primary care networks and fee for service models.

One plausible explanation for our results, given the universal access to hospital and physician services under the Canada Health Act and the well established primary healthcare system in Canada, is that the patients in the practices included in our study were 


\section{WHAT IS ALREADY KNOWN ON THIS TOPIC}

Community dwelling older adults may have unmet health needs that increase their risk of functional decline and admission to hospital or an institution

Findings from systematic reviews of preventive interventions for older adults have been inconsistent

\section{WHAT THIS STUDY ADDS}

A preventive primary care outreach intervention for older Canadian adults at risk of functional decline had no effect on QALYs, costs of health and social services, functional status, self

rated health, or mortality

The intervention had no differential effect on QALYs for patients with high or low risk of functional decline

Insufficient evidence exists to justify widespread adoption of this intervention for this target population of older adults primary healthcare system in place. Future research could also assess other patient related variables such as depression, as this is one of the best predictors of negative outcomes in older adults.

We are indebted to the research team, family physicians, and patients who participated in this study. We also thank Gary Foster, who assisted with the data management and statistical analysis.

Contributors: All authors were involved in the study concept and design and in obtaining funding. JP, KB, and CHG were involved in acquisition of data. JP, KB, CHG, and WF analysed and interpreted the data. All authors drafted the manuscript and critically revised it for important intellectual content. JP and KB supervised the study and are the guarantors. Funding: This study was supported by a grant from the Ontario Ministry of Health and Long Term Care, Primary Health Care Transition Fund. The views expressed in this paper are the views of the authors and do not necessarily reflect those of the Ontario Ministry of Health and Long Term Care. JP was supported by a Canadian Institutes of Health Research/St Joseph's Healthcare Hamilton investigator award while conducting this study. The funding agency played no role in the design and conduct of the study; in the collection, management, analysis, and interpretation of the data; or in the preparation, review, or approval of the manuscript. The researchers were independent from the funders.

Competing interests: CHG was paid as a consultant to help in developing the Health Utilities Index Mark 3 quality of life measure. WF has a stock interest in Health Utilities, which distributes copyright Health Utilities Index instrumentation and provides methodological advice on the use of Health Utilities Index.

Ethics approval: The study was approved by the Hamilton Health Sciences/McMaster Faculty of Health Sciences Research Ethics Board. Participants gave written informed consent before taking part in the study.

Data sharing: No additional data available. study may have already been providing conpr hensive care to their patients and the addition of the nurse led intervention may thus have had limited potential to provide additional benefit. Although we may have expected that the intervention would lead to increased use of physiotherapy, social work, nutritionist, and homemaker services in the intervention group, results indicate similar usage in the control group, suggesting that the control group was already receiving appropriate levels of services through the healthcare system.

The baseline Health Utilities Index Mark 3 score of 0.53 for all patients is similar to the utility score of 0.538 found for adults with stroke and much lower than the score of 0.75 found for a general population of adults aged 75 to 89 years, ${ }^{3031}$ indicating important health related quality of life deficits among our study group. This supports the ability of the Sherbrooke postal questionnaire to identify patients with important health related quality of life deficits. ${ }^{16}$

Controversy exists in the literature as to whether such interventions result in better outcomes for older, more frail adults compared with younger, less frail people. $^{7}$ The post hoc analysis of QALYs found no significant effect of the intervention for patients with either high or low Sherbrooke postal questionnaire scores for risk of functional decline.

\section{Conclusions and policy implications}

On the basis of our findings and the related literature, insufficient evidence exists to justify widespread adoption of this intervention in primary health care for this target population of older Canadian adults. Future research could examine the impact of this intervention in other countries, as Canada has a well established
1 Bergman $\mathrm{H}$, Béland F, Perrault A. The global challenge of understanding and meeting the needs of the frail older population. Aging Clin Exp Res 2002;14:223-5.

2 Coleman EA. Challenges of systems of care for frail older persons: the United States of America experience. Aging Clin Exp Res 2002;14:233-8.

3 Byles JE. A thorough going over: evidence for health assessments for older persons. Aust N Z J Public Health 2000;24:117-23.

4 Elkan R, Kendrick D, Dewey M, Hewitt M, Robinson J, Blair M, et al. Effectiveness of home based support for older people: systematic review and meta-analysis. BMJ 2001;323:1-9.

5 Stuck AE, Siu AL, Wieland GD, Adamis J, Rubenstein LZ. Comprehensive geriatric assessment: a meta-analysis of controlled trials. Lancet 1993;342:1032-6.

6 Markle-Reid M, Browne G, Weir R, Gafni A, Roberts J, Henderson SR. The effectiveness and efficiency of home-based nursing health promotion for older people: a review of the literature. Med Care Res Rev 2006;63:531-69.

7 Stuck EA, Egger M, Hammer A, Minder CE, Beck JC. Home visits to prevent nursing home admission and functional decline in elderly people: systematic review and meta-regression analysis. JAMA 2002;287:1022-8

8 Van Haastregt JCM, Diederiks JPM, van Rossum E, de Witte LP, Crebolder HFJM. Effects of preventive home visits to elderly people living in the community: systematic review. BMJ 2000;320:754-8.

9 Winograd CH. Targeting strategies: an overview of criteria and outcomes. JAGS 1991;39:25-35S.

10 Rubenstein LZ, Goodwin M, Hadley E, Patten SK, Rempusheski VF, Reuben $\mathrm{D}$, et al. Working group recommendations: targeting criteria for geriatric evaluation and management research. JAGS 1991:39:37-41S.

11 Egger M. Commentary: when, where, and why do preventive home visits work? BMJ 2001;323:8-9.

12 Ploeg J, Feightner J, Hutchison B, Patterson C, Sigouin C, Gauld M. Effectiveness of preventive primary care outreach interventions aimed at older people: meta-analysis of randomized controlled trials. Can Fam Physician 2005;51:1244-5.

13 Hébert R. Functional decline in old age. CMA/ 1997;157:1037-45

14 Hirdes JP, Naus PJ, Young JE. The use of preventive home visits among frail elderly persons: evidence from three European countries. Can J Aging 1994:13:499-509.

15 Ontario Ministry of Health and Long-Term Care. Evaluation of primary care reform pilots in Ontario phase 1-final report. 2001. www.oma. org/phealth/pcrphas11.htm.

16 Hébert R, Bravo G, Korner-Bitensky N, Voyer L. Predictive validity of a postal questionnaire for screening community-dwelling elderly 
individuals at risk of functional decline. Age Ageing 1996;25:159-67.

17 Rand Corporation. A million random digits with 100,000 normal deviates. Free Press, 1955.

18 Morris JN, Bernabei R, Ikegami N, Gilgen R, Frijters D, Hirdes JP, et al. RAl-home care (RAI-HC) manual. Canadian version. 2nd ed. Canadian Institute for Health Information, 2002.

19 Feeny D. The Health Utilities Index: a tool for assessing health benefits. Patient Reported Outcomes Newsletter 2005;34:2-6.

20 Feeny D. Preference-based measures: utility and quality-adjusted life years. In: Fayers P, Hays R, eds. Assessing quality of life in clinical trials. 2nd ed. Oxford University Press, 2005.

21 Feeny D, Farris K, Côté I, Johnson JA, Tsuyuki RT, Eng K. A cohort study found the RAND-12 and Health Utilities Mark 3 demonstrated construct validity in high-risk primary care patients. J Clin Epidemiol 2005;58:138-41.

22 Feeny D, Furlong W, Torrance GW, Goldsmith CH, Zhu Z, DePauw S, et al. Multiattribute and single-attribute utility functions for the Health Utilities Index Mark 3 system. Med Care 2002;40:113-28.

23 Furlong WJ, Feeny DH, Torrance GW, Barr RD. The Health Utilities Index (HUI) system for assessing health-related quality of life in clinical studies. Ann Med 2001;33:375-84.

24 Horsman J, Furlong W, Feeny D, Torrance G. The Health Utilities Index (HUI囚): concepts, measurement properties and applications. Health Qual Life Outcomes 2003;1:54.

25 Torrance GW, Feeny D. Utilities and quality-adjusted life years. Int J Technol Assess Health Care 1989;5:559-75.

26 Canadian Agency for Drugs and Technologies in Health. Guidelines for the economic evaluation of health technologies: Canada. 3rd ed CADTH, 2006 (available at http://cadth.ca/media/pdf/ 186_EconomicGuidelines_e.pdf).

27 Miller JD, Malthaner RA, Goldsmith CH, Goeree R, Higgins D, Cox G, et al. A randomized clinical trial of lung volume reduction surgery versus best medical care for patients with advanced emphysema: a two-year study from Canada. Ann Thorac Surg 2006;81:314-21.

28 Raynauld JP, Torrance GW, Band PA, Goldsmith CH, Tugwell P, Walker $\mathrm{V}$, et al. A prospective, randomized, pragmatic, health outcomes trial evaluating the incorporation of hylan G-F 20 into the treatment paradigm for patients with knee osteoarthritis (part 1 of 2): clinical results. Osteoarthritis Cart 2002;10:506-17.

29 Vickrey BG, Mittman BS, Connor KI, Pearson ML, Penna RDD, Ganiats TG, et al. The effect of a disease management intervention on quality and outcomes of dementia care: a randomised controlled trial. Ann Intern Med 2006;145:713-26.

30 Grootendorst P, Feeny D, Furlong W. Health Utilities Index Mark 3: evidence of construct validity for stroke and arthritis in a population health survey. Med Care 2000;38:290-9.

31 Fryback DG, Dunham NC, Palta M, Hanmer J, Buechner J, Cherepanov D, et al. US norms for six generic health-related quality of-life indexes from the National Health Measurement Study. Med Care 2007;45:1162-70.

32 Wexler DJ, Grant RW, Wittenberg E, Bosch JL, Cagliero E, Delahanty L, et al. Correlates of health-related quality of life in type 2 diabetes. Diabetologia 2006;49:1489-97.
33 Browne G, Gafni A, Roberts J, Whittaker S, Wong M, Prica M. The health and social service utilization survey 2001 for the SystemLinked Research Unit (SLRU) on health and social service utilization. McMaster University, 2001.

34 Duke University Centre for the Study of Aging and Human Development. Multidimensional functional assessment-the OARS methodology: a manual. 2nd ed. Duke University, 1978.

35 Ware JE Jr, Sherbourne CD. The MOS 36-item Short-Form Health Survey (SF-36): I. Conceptual framework and item selection. Med Care 1992;30:473-81.

36 Ware JE Jr. SF-36 Health Survey: manual and interpretation guide. Health Institute, New England Medical Centre, 1993.

37 Dupont WD, Plummer WD Jr. Power and sample size calculations: a review and computer program. Control Clin Trials 1990;11:116-28.

38 Browne G, Gafni A, Roberts J, Whittaker S, Wong M, Prica M. Approach to the measurement of costs (expenditures) when evaluating health and social programmes: the health and social service utilization survey 2001 for the System-Linked Research Unit (SLRU) on health and social service utilization. Working paper 01-4. McMaster University, 2001.

39 Ontario Ministry of Health and Long-Term Care. Ontario drug benefit formulary/comparative drug index no 39. Publications Ontario, 2005.

40 SAS Institute. Kolmogorov-Smirnov test and Kuiper test. SAS 9.2 documentation. SAS Institute, 2008.

41 Donner A, Klar N. Design and analysis of cluster randomization trials in health research. Arnold, 2000.

42 Little RJA, Rubin DB. Statistical analysis with missing data. 2nd ed. Wiley, 2002.

43 Dalby DM, Sellors JW, Fraser FD, Fraser C, van Ineveld C, Howard M. Effect of preventive home visits by a nurse on the outcomes of frail elderly people in the community: a randomized controlled trial. CMAJ 2000;162:497-500.

44 Hall N, De Beck P, Johnson D, Mackinnon K, Gutman G, Glick N. Randomized trial of a health promotion program for frail elders. Can J Aging 1992;11:72-91.

45 Hébert R, Robichaud L, Roy P, Bravo G, Voyer L. Efficacy of a nurse-led mulidimensional preventive programme for older people at risk of functional decline: a randomized controlled trial. Age Ageing 2001;30:147-53.

46 Hay WI, van Ineveld C, Browne G, Roberts J, Bell B, Mills M, et al. Prospective care of elderly patients in family practice: is screening effective? Can Fam Physician 1998;44:2677-87.

47 Bouman A, van Rossum E, Nelemans P, Kempen GIJM, Knipschild P. Effects of intensive home visiting programs for older people with poor health status: a systematic review. BMC Health Serv Res 2008;8:74.

48 Tu K, Cauch-Dudek K, Chen Z. Comparison of primary care physician payment models in the management of hypertension. Can Fam Physician 2009;55:719-27.

Accepted: 22 December 2009 